\title{
POR QUE DEVEMOS NOS IMPORTAR COM OS COLÊMBOLOS EDÁFICOS?
}

\author{
Why should we care about edaphic springtails?
}

Luís Carlos Iuñes Oliveira Filho ${ }^{1 *}$, Dilmar Baretta ${ }^{2}$

\author{
1. Professor do curso de Agronomia da Universidade do Oeste de Santa Catarina \\ (Unoesc), Campus Xanxerê - SC, E-mail: iunes1981@gmail.com (*autor para \\ correspondência). \\ 2. Professor do curso de Zootecnia da Universidade do Estado de Santa \\ Catarina (UDESC Oeste), Campus Chapecó - SC. Bolsista em Produtividade \\ Científica CNPq. E-mail: dilmar.baretta@udesc.br
}

Artigo enviado em 26/08/2016, aceito em 03/10/2016 e publicado em 20/12/2016.

\begin{abstract}
RESUMO: Este trabalho de revisão tem como objetivo apresentar a importância dos colêmbolos edáficos, com destaque para aspectos agronômicos e ecológicos. São abordadas as características gerais, densidade e distribuição dos colêmbolos, bem como a relação dos colêmbolos com práticas agrícolas, com fungos, com ciclagem de nutrientes e fertilidade do solo. São também reportados trabalhos da literatura, demonstrando a importância desses organismos aos serviços do ecossistema, como ciclagem de nutrientes, melhoria na fertilidade, agregação do solo, controle de fungos e indicadores da qualidade do solo. Pretende-se com este trabalho demonstrar o importante papel desempenhado pelos colêmbolos e expandir o campo de pesquisa com esses organismos, aumentando o conhecimento dos importantes processos mediados por eles e a interface entre a Ecologia do Solo e Ciência do Solo.
\end{abstract}

Palavras-Chave - Mesofauna do solo. Microartrópodes. Serviços do ecossistema.

\begin{abstract}
The current research aims to introduce the importance of springtails edaphic, with particular reference to agronomic and ecological aspects. General characteristics, density and distribution of springtails, and the relationship of Collembola with agricultural practices, fungi, nutrient cycling and soil fertility were covered in this study. Moreover, some literature data have been reported to demonstrate the importance of these organisms to ecosystem services such as nutrient cycling, improved fertility, soil aggregation, fungus control and indicators of soil quality. This research aims demonstrate the important role of springtails and expand the investigation field with these organisms, increasing knowledge of the important processes mediated by them and the interface between the Soil Ecology and Soil Science.
\end{abstract}

Keywords - Soil mesofauna. Microarthropod. Ecosystem services.

\section{INTRODUÇÃO}

Habitats do solo variam em tamanho de micro-nichos para paisagens inteiras, enquanto a biodiversidade do solo inclui todas as variedades de vida que habitam o habitat abaixo e acima do solo. Hoje é reconhecido que a biodiversidade do solo fornece muitos serviços ecossistêmicos, os quais desempenham um papel significativo na determinação das propriedades químicas, físicas e biológicas do solo, essenciais para os seres humanos e os ecossistemas (BREVIK et al., 2015).

A biodiversidade edáfica é um componente importante do ecossistema terrestre contribuindo com importantes serviços, como aumento da decomposição da matéria orgânica (ROŹEN et al., 2010), regulação da atividade da estrutura da comunidade (fauna do solo e microrganismos) (PEY et al., 2014, ROŹEN et al., 2010), controle de temperatura através da regulação dos fluxos de C e N (BREVIK et al., 2015), transformação e sequestro de carbono (PEY et al., 2014), dinâmica de nutrientes do solo (BREVIK et al., 2015, TEUBEN; VERHOEF, 1992), mineralização de compostos orgânicos em sais orgânicos requeridos pelas plantas superiores (RUSEK; MARSHALL, 2000), na estruturação do solo (PEY et al., 2014; SIDDIKY et al., 2012) e descontaminação do ambiente (BREVIK et al., 2015).

Devido à dependência da estrutura da comunidade biológica do solo e da atividade sobre a 
estabilidade das propriedades abióticas e bióticas do solo, qualquer mudança nestas condições pode precipitar uma mudança na biodiversidade. A mudança climática, mudança no uso e manejo do solo, poluição, espécies invasoras e qualquer fator que contribui para a degradação do solo podem afetar a biodiversidade (BREVIK et al., 2015). Mudanças nas comunidades do solo e a perda de biodiversidade do solo ameaçam a multifuncionalidade e sustentabilidade dos ecossistemas (WAGG et al., 2014).

Assim, Collembola é um grupo de microartrópodes pertencentes a mesofauna e amplamente distribuídos no solo (GANGE; BOWER, 1997) e são excelentes representantes da diversidade da fauna do solo (CASSAGNE et al., 2003). Estes microartrópodes do solo têm influência significativa na ecologia microbiana do solo, ciclagem de nutrientes e fertilidade do solo, alimentando-se de microrganismos do solo e matéria orgânica morta. Por sua vez eles também respondem a uma variedade de fatores ambientais e ecológicos, como mudanças na química do solo, configuração do microhabitat e práticas agrícolas (HOPKIN, 1997). Sendo assim considerados bons indicadores de qualidade do solo (BARETTA et al., 2008; BARTZ et al., 2014; PARISI et al., 2005, PONGE et al., 2003, 2006; SOUSA et al., 2006).

Esse texto não pretende ser uma fonte completa de informações sobre a Classe Collembola e sim uma breve revisão sobre o papel desempenhado pelos colêmbolos em alguns aspectos de importância ecológica, voltado tanto para Agronomia como para Ciência do Solo, bem como trazer trabalhos realizados no Brasil e no exterior. Examinamos os aspectos estruturais - características gerais, distribuição e densidade dos colêmbolos e, então, nos voltamos para seus aspectos funcionais, aspectos agronômicos e ecológicos. Obviamente, por razões práticas muitas publicações não foram consideradas nesse artigo.

\section{DESENVOLVIMENTO}

\section{Características gerais dos colêmbolos}

Collembola constitui um grupo extremamente antigo, mas sua posição exata dentro de Arthropoda ainda é bastante discutida, pois os colêmbolos foram por muito tempo considerado da Classe Insecta. Nessa revisão, optou-se a classificação proposta por Zhang (2011) em que o grupo Collembola é considerado como Classe dentro de Arthropoda.

A Classe Collembola é representada por quatro ordens distintas que até o momento são as mais aceitas e está distribuída em Poduromorpha, Entomobryomorpha, Neelipleona e Symphypleona (JANSSENS; CHRISTIANSEN, 2011). As ordens Poduromorpha e Entomobryomorpha, são mais longos e largos e as divisões entre os segmentos torácicos e abdominais são facilmente visíveis, enquanto que Neelipleona e Symphypleona apresentam o corpo globoso com fusão dos segmentos (ZEPPELINI FILHO; BELLINI, 2004). Alguns autores, ainda distinguem uma nova ordem, Metaxypleona, com posição filogenética indefinida (ZEPPELINI, 2012).

De modo geral, os colêmbolos, em função da espécie, apresentam um comprimento do corpo entre 1 e $5 \mathrm{~mm}$, algumas espécies apresentam tamanho de 0,25 $\mathrm{mm}$, Megalothorax minimus e no máximo $17 \mathrm{~mm}$ como Holocanthella duospinosa (BELLINGER et al., 2016). A coloração dos colêmbolos é bastante variável, sendo que espécies que habitam locais mais escuros como cavernas, na interface solo-serapilheira e até mesmo no solo tendem a ser brancos ou cinzentos enquanto as que vivem em ambientes mais abertos apresentam uma escala mais colorida (HUTCHINS et al., 2004). São ápteros e ametábolos, sendo assim, não apresentam metamorfose, realizando mudas durante toda a vida (ZEPPELINI FILHO; BELLINI, 2004).

Vários estudos foram realizados sobre a escolha de alimento por colêmbolos, os quais explicam que a alimentação é influenciada pela preferência e disponibilidade do alimento, hábitat, tamanho do aparelho bucal e atividade enzimática (NAKAMORI; SUZUKI, 2008). Os colêmbolos podem alimentar-se de uma gama extensiva de alimentos, mas os microrganismos e hifas de fungos, associadas à matéria orgânica do solo e serapilheira são os alimentos preferidos da maioria das espécies (FOUNTAIN; HOPKIN, 2005; HOPKIN, 1997; ZEPPELINI FILHO; BELLINI, 2004). Alguns grupos alimentam-se de fezes de outros invertebrados de solo (CHRISTIANSEN, 1970), substância vegetal ou animal em decomposição, de algas e de liquens, material vegetal fresco (principalmente família Sminthuridae), sendo também encontrado pólen no intestino de algumas espécies (DALY et al., 1998). Ainda, indivíduos Folsomia candida foram encontrados alimentando-se do revestimento mucilaginoso de semente, bem como do embrião da Plantago major (NIETSCHKE et al., 2011).

Algumas espécies se constituem pragas, como Bourletiella hortensis, que pode danificar mudas de diversas espécies em começo de primavera e Hypogastrura armata que é uma praga frequente de cogumelos comerciais (HUTCHINS et al., 2004); Sminthurus viridis é uma importante praga das culturas 
de grãos de inverno e pastagens (ROBERTS et al., 2011). Existem espécies predadoras, alimentando-se de nematóides, pois várias espécies de nematóides têm sido encontradas no intestino de algumas espécies de Entomobrya sp. (HUTCHINS et al., 2004). Em densidades muito altas há referências de canibalismo (DALY et al., 1998). Rotheray et al. (2009) relatam que em florestas temperadas os colêmbolos alimentam-se predominantemente de detritos vegetais e fungos. A preferência alimentar por diferentes fungos (por exemplo, Hypholoma fasciculare, Phallus impudicus, Phanerochetae velutina e Resinicium bicolor) reflete no valor do recurso em termos de crescimento e reprodução dos colêmbolos.

\section{Distribuição e densidade}

A Classe Collembola é um grupo de microartrópodes pertencentes à mesofauna $\mathrm{e}$ amplamente distribuídos no solo (GANGE; BOWER, 1997), apresentando distribuição mundial, desde O círculo polar Ártico até a Antártida (ZEPPELINI FILHO; BELLINI, 2004) e sobrevivendo em temperaturas de $-60^{\circ} \mathrm{C}$ (HUTCHINS et al., 2004). São conhecidas espécies vivendo em montanhas com 5.000 $\mathrm{m}$ acima do nível do mar e outras em partes profundas de cavernas, tanto em clima tropical quanto temperado (ZEPPELINI, 2012).

Em levantamento recente do número de espécies descritas, Bellinger et al. (2016) relatam 8.603 espécies descritas. Os autores ainda fazem um resumo do número de espécies descritas no mundo por ano, o qual é atualizado constantemente desde 1758 (ver mais detalhes

http://www.collembola.org/doc/species.htm).

Também foi publicada uma lista dos colêmbolos encontrados no Brasil, sendo 287 espécies distribuídas em 94 gêneros e 19 famílias (ABRANTES et al., 2012). Tomando como base a estimativa de que ainda faltam descrever cerca de 50.000 espécies de colêmbolos (HOPKIN, 1998), ainda há trabalho para os taxonomistas atuais por aproximadamente 492 anos (BELLINGER et al., 2016).

Os colêmbolos são capazes de viver em uma diversidade de habitats, desde solo e micro hábitats adjacentes com abundância de matéria orgânica, serapilheira, passando por ninhos de insetos sociais (formigueiros e termiteiros), musgos, bainha de folhas de plantas, dossel de florestas tropicais, espaços aéreos da areia em dunas e praias, cavernas, desertos na Austrália, embora menos abundantes em regiões secas, até superfície de lagos de água doce ou ao longo de praias marinhas (HUTCHINS et al., 2004;
ZEPPELINI FILHO; BELLINI, 2004; ZEPPELINI, 2012). Em certos hábitats, como em alguns locais de pastagem, as densidades podem exceder 750 milhões de indivíduos (ind.) por hectare (HUTCHINS et al., 2004).

A maioria dos colêmbolos é encontrada até uma profundidade de $10 \mathrm{~cm}$, a grande maior parte está na serapilheira e primeiros centímetros do solo, dependendo de tipo de hábitat, embora algumas espécies sejam distribuídas uniformemente dentro do perfil (CHRISTIANSEN, 1964), tendo sido encontradas espécies (Folsomia fimetaria, Isotoma anglicana, Isotama notabilis, Heteromurus nitidus, Protaphorura armata, Pseudosinella alba) até $32 \mathrm{~cm}$ de profundidade (JØRGENSEN et al., 2003). Assim, são identificadas três formas de vida (morfotipos) típicas de colêmbolos consoante com seu grau de adaptação ao solo com base na morfologia e habitat, classificados como epigeicos (epiedáfico), hemiedáficos e edáficos (euedáfico) (Figura 1) (GISIN, 1943; PETERSEN, 2002). Edáficos e epigeicos são opostos, enquanto a forma de vida hemiedáfica é considerada intermediária (PETERSEN, 2002). Espécies epigeicas têm uma alta atividade metabólica, consomem um substrato de alta qualidade (GISIN, 1943) movem ativamente na superfície do solo, dentro da camada de serapilheira e sobre a vegetação (QUERNER et al., 2013). As espécies edáficas consomem alimentos de baixa qualidade e têm uma atividade metabólica baixa (GISIN, 1943). Juntos, hemiedáficos ( $5 \mathrm{~cm}$ da superfície do solo) e edáficas (camada de solo mais profundo, inferior a $5 \mathrm{~cm}$ ), são agrupados em forma de vida endogeica (PETERSEN, 2002; QUERNER et al., 2013). Espécies endogeicas são encontradas no interior dos poros do solo e são influenciadas mais pelos parâmetros do local, porque vivem dentro dos poros do solo, são menores e menos móveis (QUERNER et al., 2013).

A densidade de colêmbolos é extremamente variável dependendo do hábitat, estação e atividade humana (BAKONYI et al., 2002). No geral, em diferentes solos a densidade de colêmbolos está entre $10^{2}$ e $6,7 \times 10^{5}$ ind. $\mathrm{m}^{-2}$ (GILLER, 1996). Em solos agrícolas as densidades de colêmbolos variam de 10.000 a 120.000 ind. $\mathrm{m}^{-2}$ (FRAMPTON; VAN DEN BRINK, 2007; LARSEN et al., 2004). Na maioria dos solos temperados suas densidades são de $10^{4}-10^{5}$ ind. $\mathrm{m}^{-2}$ (PETERSEN; LUXTON, 1982). Contudo, em solos agrícolas de clima temperado geralmente são encontradas baixas densidades de colêmbolos (menos de 20.000 ind $\mathrm{m}^{-2}$ ) (DITTMER; SCHRADER, 2000; WINTER et al., 1990). Porém, em solos tropicais especificamente no Brasil (Tabela 1), densidades altas de Collembola, em média 60.600 ind. $\mathrm{m}^{-2}$, foram 
encontradas em alguns solos agrícolas (CULIK et al., 2002) e de 14.000 a 56.000 ind. $\mathrm{m}^{-2}$ em solos de pomares de mamão (CULIK et al., 2006). Apesar do grande número de indivíduos por metro quadrado e de seu tamanho pequeno a contribuição de colêmbolos para biomassa animal e respiração do solo é baixa, tipicamente entre 1 e $5 \%$ em ecossistemas temperados, mas até aproximadamente 10\% em alguns locais árticos e até $33 \%$ do total da respiração da fauna do solo em ecossistemas em fases iniciais de sucessão (HOPKIN, 2002).

Comparar os valores encontrados na literatura é difícil, pois as metodologias utilizadas são bastante variáveis entre os estudos, especialmente quanto à profundidade de amostragem (CLUZEAU et al., 2012). Além disso, diversos fatores podem influenciar a composição, a densidade e a atividade das espécies, como as condições climáticas, as propriedades do solo (tipo de solo, estrutura, $\mathrm{pH}$, teor de matéria orgânica, etc.) e a composição de espécies de organismos (plantas, microrganismos e fauna do solo) (PFLUG; WOLTERS, 2002; ROŹEN et al., 2010; SCHRÖDER, 2008) e o período de amostragem (CLUZEAU et al., 2012).
A variação da densidade das populações de colêmbolos está, em geral, relacionada com fatores ecológicos que alteram a sua atividade. Jucevica e Melecis (2006) mostraram através de uma análise dos efeitos em longo prazo do aquecimento climático, em solo com pinheiros da zona temperada, que várias formas de vida ecomorfológicas de Collembola podem diferir com às mudanças climáticas. Espécies edáficas são mais afetadas pelo aumento da temperatura, enquanto que as hemiedáficas parecem ser mais sensíveis às condições de umidade. Deve ser enfatizado que as respostas da comunidade são fortemente controladas por temperatura e precipitação (JUCEVICA; MELECIS, 2006), sendo os colêmbolos dependentes de um fornecimento de água, com possíveis migrações verticais e horizontais no solo para encontrarem as condições mais adequadas. A falta de umidade pode aumentar a taxa de mortalidade em espécies mais sensíveis, situação que também afeta a comunidade fúngica do solo, importante fonte de alimento para os colêmbolos.

Tabela 1. Densidade de colêmbolos em trabalhos realizados no Brasil.

\begin{tabular}{|c|c|c|}
\hline Local/Data - Solo/Área/Bioma/Tratamento & Método de coleta & $\begin{array}{l}\text { Densidades } \\
\text { colêmbolos }\end{array}$ \\
\hline $\begin{array}{l}\text { BARETTA et al. (2008) }{ }^{1} \\
\text { São Paulo / Setembro de } 2004 \\
\text { Latossolo Vermelho-Amarelo distrófico } \\
\text { Floresta nativa com predominância de araucária } \\
\text { (Araucaria angustifolia) (A); Reflorestamento de } \\
\text { araucária (B); Reflorestamento de araucária } \\
\text { submetido a incêndio acidental (C); Pastagem } \\
\text { natural com araucárias nativas e ocorrência de } \\
\text { incêndio acidental (D). }\end{array}$ & $\begin{array}{l}\text { "Trampas de Tretzel" (Pitfall } \\
\text { traps, com } 6 \mathrm{~cm} \text { de diâmetro), } \\
\text { permanecendo no campo por } \\
\text { três dias. }\end{array}$ & 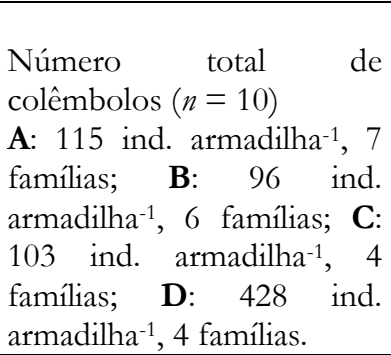 \\
\hline $\begin{array}{l}\text { ROVEDDER et al. (2009) } \\
\text { Rio Grande do Sul / Fevereiro e maio de } 2006 \\
\text { Neossolo Quartzarênico } \\
\text { Solo com cobertura natural de Lupinus albescens } \\
\text { (A); Área arenizada revegetada com L. albescens há } \\
\text { três anos (B); Área arenizada revegetada com L. } \\
\text { albescens há um ano (C); Campo nativo (D); Solo } \\
\text { degradado por arenização há } 20 \text { anos (E). }\end{array}$ & $\begin{array}{l}\text { Armadilhas do tipo PROVID } \\
\text { (Pitfall traps) permanecendo } \\
\text { no campo por quatro dias. }\end{array}$ & 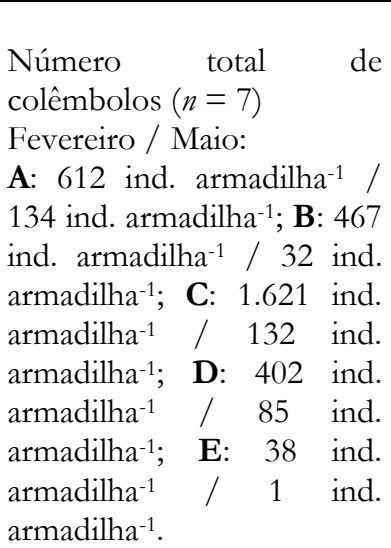 \\
\hline
\end{tabular}

ZEPPELINI et al. (2009) ${ }^{2}$

Paraíba / Novembro de 2005 e abril de 2006

Armadilhas do tipo Malaise

Número

total de 
Toda a área originalmente foi coberta por Restinga. Esta vegetação ainda está presente em áreas adjacentes não perturbadas. $\mathrm{O}$ processo de mineração envolve a remoção de toda a vegetação e do processamento da areia para extrair minerais de interesse.

Quatro áreas reflorestadas com 2, 4, 8 e 16 anos e uma área controle (não perturbada, em que nenhum desmatamento, caça ou outra exploração tenha sido autorizado nos últimos 30 anos).

\section{DRESCHER et al. (2011) 3}

Rio Grande do Sul / Fevereiro de 2008

Neossolo Litólico

Fumicultura em Plantio Direto (PD); Fumicultura em Preparo Convencional (PC); Fumicultura em Cultivo Mínimo (CM); Reconversão para Vitivinicultura (RV); Mata Nativa (MN).

CULIK et al. (2002)

Espirito Santo / Dezembro de 1999 e novembro de 2000

Adubação orgânica (15.000 kg composto seco ha 1), Latossolo (A1); Adubação inorgânica (tendo como base a necessidade da cultura), Latossolo (A2); Plantio direto, Latossolo (B1); Convencional, Latossolo (B2); Cobertura morta (casca de arroz ou grama picada aplicado a uma profundidade de 2-3 cm no plantio), Aluvial (C1); Sem cobertura, Aluvial (C2). permanecendo no campo por sete dias.

Armadilhas do tipo PROVID (Pitfall traps) permanecendo no campo por quatro dias.

Cilindro $(4,8 \mathrm{~cm}$ de diâmetro, $10 \mathrm{~cm}$ profundidade).

colêmbolos $(n=30)$

2 anos: 108 ind. armadilha 1, 1 espécie; 4 anos: 647 ind. armadilha ${ }^{-1}, 5$ espécies; 8 anos: 2.937 ind. armadilha ${ }^{-1}, 7$ espécies; 16 anos: 3.907 ind. armadilha 1, 11 espécies; Controle: 4.584 ind. armadilha ${ }^{-1}, 19$ espécies.

Número total de colêmbolos $(n=10)$

PD: 1.389 ind. armadilha ${ }^{-1}$; PC: 1.054 ind. armadilha ${ }^{-1}$; CM: 1.273 ind. armadilha ${ }^{-1}$; RV: 1.074 ind. armadilha ${ }^{-1}$; MN: 618 ind. armadilha ${ }^{-1}$.

Número total de colêmbolos (local A, $n=$ 16; local B e C, $n=14$ )

A1: 68.525 ind. $\mathrm{m}^{-2}, 24$ espécies; A2: 46.731 ind. $\mathrm{m}-$ 2, 23 espécies; B1: 56.052 ind. $\mathrm{m}^{-2}, 27$ espécies; B2: 32.999 ind. $\mathrm{m}^{-2}, \quad 25$ espécies; C1: 103.656 ind. m², 27 espécies; C2: 56.486 ind. $\mathrm{m}^{-2}, 22$ espécies.

Total de 38 espécies coletadas.

DUARTE (2004) 5

Rio Grande do Sul / Junho de 2000

Fragmento de mata com araucária $\mathbf{A 1}$ (Estação Ecológica de Aracuri), camada de serapilheira 1$3 \mathrm{~cm}$; Fragmento A2 (Estação Ecológica de Aracuri), maior que A1, camada de serapilheira 1-3 cm; Fragmento M1, mata ciliar do rio Saltinho, camada de serapilheira cerca de $1,0 \mathrm{~cm}$; Fragmento M2, nascente de um pequeno riacho, que corre para o rio Saltinho, camada de serapilheira entre 1-2 cm; Fragmento M3, áreas de intenso pisoteio pelo gado, serapilheira escassa $(<1 \mathrm{~cm})$; Fragmento M4, mais aberto e com estrato arbóreo alterado, serapilheira mais escassa $(<0,5 \mathrm{~cm})$; Fragmento M5, camada de serapilheira 1-2 cm.

CULIK et al. $(2006)^{6}$

Espirito Santo / Intervalos de três meses à partir de fevereiro de 2003 até novembro de 2003

Fazenda Experimental de Sooretama, pomar de
Amostra $(7 \mathrm{~cm}$ de diâmetro, $6 \mathrm{~cm}$ profundidade, serapilheira + solo).

Extração durante sete dias em extratores tipo BerleseTullgren modificados.
Densidade média $(n=9)$

A1: 7.080 ind. $\mathrm{m}^{-2} ; \mathbf{A 2}$ : 15.851 ind. $\mathrm{m}^{-2}$; M1: 11.461 ind. $\mathrm{m}^{-2}$; M2: 13.541 ind. $\mathrm{m}^{-2}$; M3: 15.537 ind. $\mathrm{m}^{-2}$; M4: 15.143 ind $\mathrm{m}^{-2}$; M5: 10.355 ind. $\mathrm{m}^{-2}$.
Amostra de solo $(4,8 \mathrm{~cm}$ de Número total de diâmetro, $10 \mathrm{~cm}$ de colêmbolos profundidade) $\quad$ SO PC $(n=16): 14.000$ 
produção convencional, com irrigação por aspersão, SO PC; Pomar de produção integrada, com irrigação por gotejamento, SO PI-G; Pomar de produção integrada, com irrigação por microaspersão, SO PI-M

Pomar comercial em Linhares de produção convencional, NB PC e LB PC; Pomar comercial de produção integrada, SV PI e CA PI BARROS et al. (2010)

Paraná / Maio, setembro e novembro de 2007 e janeiro de 2008

Área de mineração e metalurgia de $\mathrm{Pb}$.

Solo de referência sob mata nativa e Panicum maximum no sub-bosque. Neossolo Litólico (A); Localizada em posição intermediária da encosta, representando condição onde ocorreu incorporação de rejeito grosseiro e de coloração escura no perfil de solo, devido à deposição de colúvio das áreas mais altas. Cambissolo Háplico (B); Localizado próximo a fábrica, com grande volume de serapilheira, com os horizontes do solo preservados e sem incorporação de rejeitos sólidos. Potencialmente contaminado com material particulado oriundo do processo de fusão do $\mathrm{Pb}$, por meio de emissão de fumaça pelas chaminés. Neossolo Litólico (C); Pilhas de rejeitos na superfície do solo e resíduos ainda frescos visivelmente incorporados no perfil. Mistura de solo mais rejeito grosseiro (D); Localizado próximo a fábrica e sujeito à contaminação atmosférica por $\mathrm{Pb}$. Neossolo Quartzarênico (E)

1 Famílias: Brachystomellidae, Entomobrydae, Hypogasturidae, Isotomidae, Katiannidae, Paronellidae, Sminthuridae e Tomoceridae.

2 Espécies: Arlesia albipes, Brachystomella cf. agrosa, Dicyrtoma sp. n. 1, Dicyrtoma sp. n. 2, Entomobrya cf. nivalis, E. grisoolivata, Hemisotoma thermophilus, Lepidocyrtus sp. n. 1, Lepidocyrtus sp. 2, Prorastriopes sp. n., Rastriopes sp. n., Seira sp. n. 1, Seira sp. n. 2, Seira sp. n. 3, Seira sp. n. 4, S. brasiliana, S. mirianae, S. prodiga, S. xinguensis, Sphaeridia sp., Sphyrotheca mucroserrata, Sminthurinus sp. n. 1, Sminthurinus sp. n. 2, Xenylla sp. n.

3 Famílias: PD: Entomobrydae, Sminthuridae, Poduridae, Hypogasturidae. PC: Entomobrydae, Sminthuridae. CM: Entomobrydae, Sminthuridae. RV: Entomobrydae, Sminthuridae. MN: Entomobrydae, Sminthuridae.

${ }^{4}$ Espécies: Ceratophysella boletivora, Hypogastrura manubrialis, Xenylla welchi, X. sp. 2, Brachystomella sp.1, Friesea sp.1, Vitronura giselae, Thalassaphorura encarpata, Mesaphorura yosiii, M. silvicola, M. simoni, Folsomides centralis, F. parvulus, Proisotoma minuta, P. tenella, Ballistura fitchi, Cryptopygus thermophilus, Desoria trispinata, Isotomiella symmetrimucronata, Isotomodes ccoccatirensis $1 \mathrm{sp}$. aff. pseudoproductus, Isotomurus bimus, Folsomia wellingdae, F. amazonae, F.onychiurina, Heteromurus sp. 1, Entomobrya sp.1, Lepidocrytus nr. mutabilis, Pseudosinella nr. biunguiculata, P. sp.2, Cyphoderus sp.1, Trogolaphysa sp.1, Campylothorax sp.1, Neelus tristani, Arrhopalites sp.1, Sminthurinus sp., S. sp.1 e sp.2 e Bourletiella sp.1.

5 Ordens: Arthropleona e Shymphypleona.

${ }^{6}$ Entomobyidae não identificado, Hypogastruridae não identificado, Isotomidae não identificado, Sminthuridae não identificado, Arrhopalites sp. 1 (sp. nov.), Ballistura fitchi, Bourletiella sp. 1, Brachystomella sp. 1 (sp. nov.), Campylothorax sp. 1, Ceratophysella boletivora, Cryptopygus thermophilus, Cyphoderus sp. 1, Desoria trispinata, Entomobrya sp. 1, Folsomia amazonae, Folsomia wellingdae, Folsomides centralis, Folsomides parvulus, Folsomina onychiurina, Friesea sp., Heteromurus sp. 1, Hypogastrura manubrialis, Isotomiella symmetrimucronata, Isotomodes ccoccatirensis 1 sp. aff. pseudoproductus, Isotomurus bimus, Lepidocrytus nr. mutabilis, Mesaphorura silvicola, Mesaphorura simoni, Mesaphorura yosiii, Neelus tristani, Proisotoma minuta, Proisotoma tenella, Pseudosinella nr. biunguiculata, Pseudosinella sp. 2, Sminthurinus sp. (juvenile), Sminthurinus sp. 1, Sminthurinus sp. 2, Thalassaphorura encarpata, Trogolaphysa sp. 1, Vitronura giselae, Xenylla sp., Xenylla welchi. 
A vegetação também influencia os colêmbolos. Zonas com um estrato herbáceo mais desenvolvido apresentam uma maior densidade de indivíduos, pois o ar encontra-se mais saturado e existem mais refúgios (BERBIERS et al., 1989). Outro fator que influencia na densidade populacional é a predação por artrópodes da serapilheira como ácaros das espécies Bdellodes lapidaria, Neomolgus capillatus) (ROBERTS et al., 2011), coleópteros, aranhas (BUZZI, 2013, DALY et al., 1998), formigas do gênero Strumigenys (Myrmicinae) (GRONENBERG, 1996), dentre outros.

A abundância de Collembola em solos cultiváveis está ligada com a estrutura do solo. Colêmbolos não podem fazer sua própria cova e são dependentes de poros cheios de ar dimensionados com pelo menos a largura de seu corpo, tendendo a evitar poros estreitos, provavelmente para proteger sua cobertura de cera contra danos (LARSEN et al., 2004). $\mathrm{O}$ ponto em que colêmbolos podem acessar e habitar os poros do solo é determinado pelos seguintes aspectos de condições físicas do solo: (1) o volume de espaço poroso habitável, (2) conectividade destes poros e (3) umidade e regimes de temperatura (DIDDEN, 1987; HOPKIN 1997; JOOSSE, 1981; LARSEN et al., 2004). Estes parâmetros, o tamanho dos colêmbolos, como eles se comportam e respondem as condições em geral do solo são fundamentais para abundância e composição de espécies (CHAGNON et al., 2000).

Um estudo feito em recipientes com Protaphorura fimata, demonstrou que juvenis não mostraram preferência por estar em solo menos denso
(9,3\% de poros maiores que $300 \mu \mathrm{m})$ ou mais denso $(5,6 \%$ de poros maiores que $300 \mu \mathrm{m})$, enquanto animais maiores mostraram uma preferência significante para solo menos denso, embora o solo mais denso contivesse espaço poroso habitável (DIDDEN, 1987). Haarløv (1960) conduziu outro estudo de inter-relações entre porosidade do solo e microartrópodes (inclusive Collembola), em condições de campo e concluiu que animais menores tendem a ser dominante em hábitats ou camadas com tamanhos de poros pequenos. Correlações entre aumento da densidade do solo e redução em abundância e diversidade de espécies de colêmbolos edáficos foram encontradas em vários estudos (DITTMER; SCHRADER, 2000; HEISLER; KAISER, 1995; SCHRADER; LINGNAU, 1997). Estes resultados mostram que a estrutura do solo e a diminuição do espaço poroso habitável são parâmetros chaves na abundância de colêmbolos edáficos. Isto sugere que o espaço poroso habitável para colêmbolos é um ambiente no qual ele é capaz de desenvolver todas as suas estruturas morfológicas características, evidenciado por diferentes formas de vida ecomorfológicos (QUERNER et al., 2013).

Outros fatores como $\mathrm{CO}_{2}$ e altitude são de extrema importância para o estabelecimento de espécies de colêmbolos, sendo o $\mathrm{CO}_{2}$ um fator importante para explicar o agrupamento de espécies, juntamente com a inclinação (relevo) e a umidade (GARCÍA-GÓMEZ et al., 2009). 


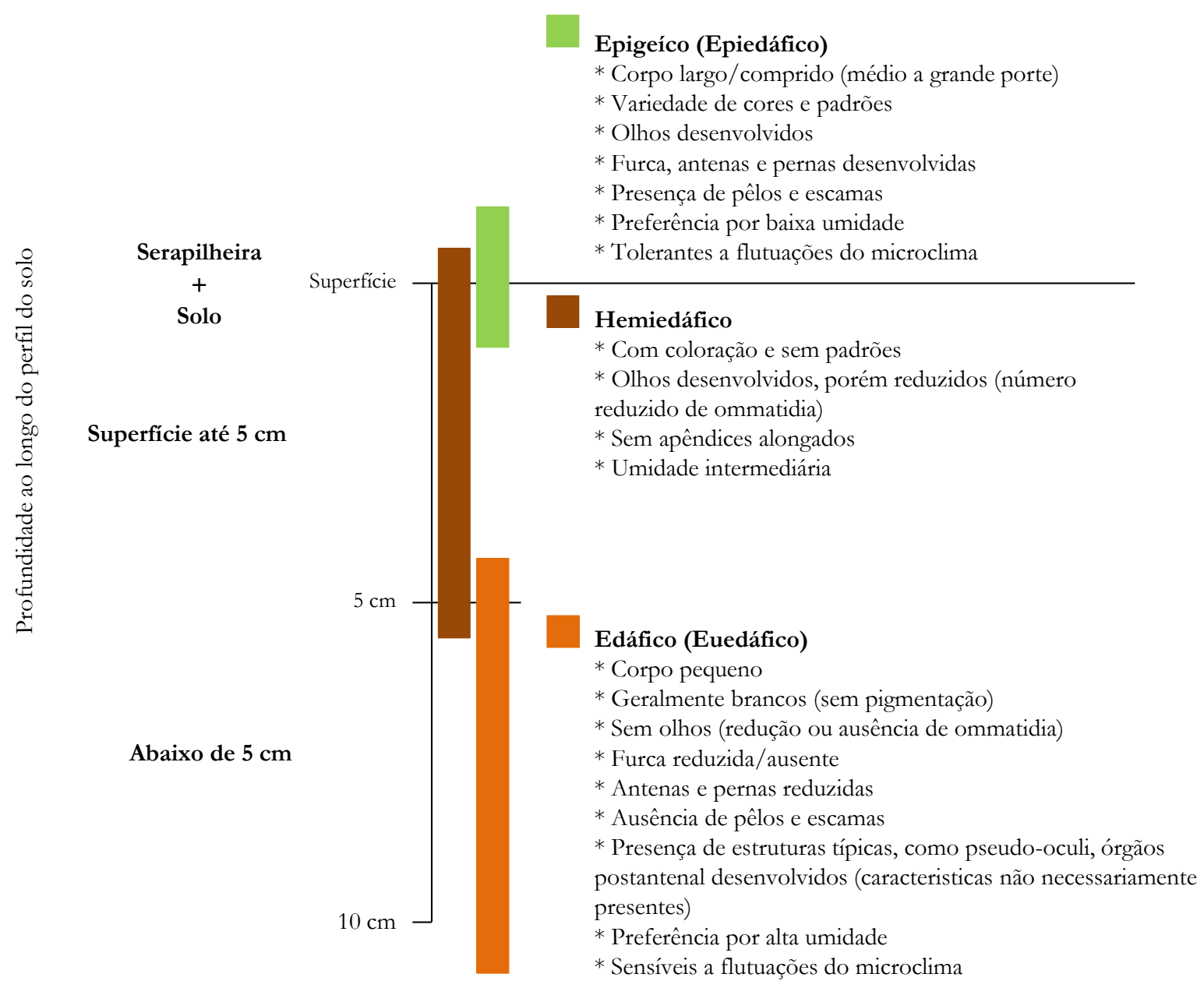

Figura 1. Mudança na forma de vida (morfotipo) de espécimes de colêmbolos habitantes do solo e das que vivem na superfície. Barras coloridas indicam a profundidade que melhor se adaptam as espécies de colêmbolos epigeicas (barra verde), hemiedáficas (marron escuro) e edáficas (marrom claro). Embora as espécies tenham uma profundidade preferencial que melhor se adaptam, elas podem por algum fator abiótico (por exemplo, umidade e temperatura) ou biótico (por exemplo, predador ou falta de alimento) migrarem verticalmente no perfil. A quantificação do quanto cada morfotipo pode migrar no perfil ainda não é bem definida. Informações adaptadas de Krab et al. (2010), Parisi et al. (2005) e Querner et al. (2013).

A sensibilidade que estes artrópodes apresentam em relação à modificação das suas condições ambientais faz da sua utilização uma importante ferramenta para o estudo da influência de fatores físico-químicos e microbiológicos sobre a fauna do solo (BARETTA et al., 2008).

\section{Aspectos agronômicos e ecológicos}

Funções e serviços dos ecossistemas terrestres apresentam uma forte dependência dos solos e da biota do solo (BROWN et al., 2015). De fato, os elementos biológicos são a chave para as funções dos ecossistemas e a identificação de bioindicadores é importante para cumprir com a proteção dos solos (HAVLICEK, 2012). Existem grupos de organismos cujos benefícios são cada vez mais estudados e conhecidos. Embora os colêmbolos tenham recebido muita atenção por parte dos pesquisadores nos últimos anos, muitos de seus benefícios não são de conhecimento público. A grande diversidade de espécies de colêmbolos resulta em diversos papéis funcionais dentro dos processos do solo (Tabela 2) que refletem nos serviços prestados aos ecossistemas. 
Tabela 2. Características de Collembola e sua potencial influência na composição da comunidade e processos do ecossistema (modificado de VAN STRAALEN et al., 2008).

\begin{tabular}{ll}
\hline Característica & Efeitos potenciais na comunidade e funcionamento do ecossistema \\
\hline $\begin{array}{l}\text { Morfológico } \\
\text { Tamanho de corpo }\end{array}$ & Utilização de recursos complementares e diversidade da comunidade \\
Biomassa & Habilidade de dispersão e distribuição em profundidade \\
Número de ocelos & Habilidade de dispersão \\
$\begin{array}{l}\text { Desenvolvimento da furca } \\
\text { Pigmentação (coloração) }\end{array}$ & Atividade em habitats abertos \\
\hline Fisiológico & Profundidade específica de atividade alimentar \\
Tolerância a seca & Distribuição geográfica \\
Tolerância ao frio & Atividade em habitat específico \\
Resistência ao calor & Resiliência, recuperação de distúrbios \\
pH ótimo & \\
Modo de reprodução & Adaptação ao estresse ambiental \\
Longevidade & Estrutura e sucessão da comunidade de plantas, estrutura da comunidade \\
Característica de plasticidade & de microrganismos, controle biológico e ciclagem de nutrientes \\
\hline Ecológico & Redução da competição, diversidade da comunidade e ciclagem de \\
Preferência alimentar & nutrientes \\
Estratificação vertical & Dispersão e distribuição geográfica \\
\hline
\end{tabular}

\section{Relação dos colêmbolos com práticas agrícolas}

Mudança do uso do solo é o fator primário determinante da diversidade dos organismos do solo em níveis local e regional (BARETTA et al., 2008; BENGTSSON, 2002; LAVELLE et al., 1997). Embora, efeitos específicos variam com táxon e local geográfico, o manejo muda a composição da comunidade, padrões de distribuição da população e diminuição da abundância global de invertebrados (EATON et al., 2004; STINNER; HOUSE, 1990). Segundo Chauvat et al. (2003), os colêmbolos são estrategistas $r$ e respondem rapidamente às mudanças ambientais.

Espécies de colêmbolos são bastante onipresentes com efeitos limitados do manejo agrícola na diversidade (BRENNAN et al., 2006; CHAUVAT et al., 2007). De acordo com Kladivko (2001), frequentemente a população de colêmbolos é diminuída que aumentada através do cultivo. Alvarez et al. (2001), avaliando as populações de colêmbolos em regimes de manejo agrícola convencional, integrado e orgânico, encontraram diferenças mínimas na composição de comunidades de colêmbolos. Baretta et al. (2006) encontraram forte associação dos sistemas de preparo convencionais, em sucessão e rotação de culturas com o aumento da densidade do grupo Collembola e diminuição da densidade da fauna edáfica, quando comparados os sistemas mais conservacionistas como plantio direto e cultivo mínimo no Planalto Sul Catarinense.

Variação da qualidade e quantidade de cobertura do solo influencia na alimentação dos organismos edáfico (BARETTA et al., 2003) sendo que maior cobertura do solo pode diminuir a variação da temperatura superficial pelo aumento do sombreamento e da umidade. A existência de um horizonte orgânico bem formado é essencial para promover microhabitat favorável à manutenção, sobretudo de colêmbolos epiedáficos e hemiedáficos, principalmente em termos de umidade e provisão de alimento (GAMA et al., 2003). De acordo com Hodkinson et al. (1994) os colêmbolos são muito sensíveis à dessecação e perda de água cuticular, o que associado à ausência de cobertura vegetal e alta temperatura do solo, resulta em uma população reduzida.

No plantio direto, a cobertura que permanece na superfície do solo é o fator provável 
que influencia as populações de colêmbolos pela criação ou manutenção de uma camada de material orgânico isolante. Resultados obtidos por Eaton et al. (2004) sugerem que os colêmbolos são sensíveis às perturbações na camada superficial de matéria orgânica. Estas perturbações podem ser direta, tal como a remoção da camada de matéria orgânica, ou indireta, tais como as mudanças no microclima e redução da qualidade ou quantidade de serapilheira, que pode ocorrer como um resultado de controle da vegetação. A densidade de microartrópodes tende a ser maior em locais com cobertura morta do que sem cobertura (BARETTA et al., 2006).

A estrutura do solo é afetada por várias propriedades e pela atividade da biota (BRONICK; LAL, 2005), sendo a agregação um importante processo do ecossistema mediado pela biota do solo. Alguns estudos têm pesquisado os efeitos dos colêmbolos na estruturação e agregação do solo (CARUSO et al., 2011; DAVIDSON; GRIEVE, 2006; SIDDIKY, et al. 2012), enquanto muitos têm quantificado os efeitos dos fungos micorrízicos arbusculares (FMAs) e minhocas sobre a sua agregação.

Siddiky et al. (2012) realizaram um estudo em casa de vegetação testando os efeitos de espécies de plantas (Sorghum vulgare e Daucus carota), presença/ausência de colêmbolos (Proisotoma minuta), presença/ausência de FMAs e suas interações. Neste estudo, o solo que foi exposto individualmente ao crescimento fúngico e colêmbolos teve maior estabilidade de agregados do solo em água quando comparado ao controle. Além disso, os autores observaram que a presença de $P$. minuta aumentou claramente a formação de macroagregados (maior que $0,25 \mathrm{~mm}$ ) em comparação aos tratamentos sem a presença de colêmbolos. Os resultados mostram que os colêmbolos podem realizar um papel crucial na sustentabilidade ecológica ao promover agregação do solo e apontam sobre a importância de considerar interações de organismos no entendimento da formação da estrutura do solo.

Outras práticas específicas de manejo como a aplicação de fertilizantes e pesticidas apresentam efeitos positivos ou negativos na abundância $\mathrm{e}$ diversidade de colêmbolos. A adubação através da adição de estercos ao solo ou combinações de esterco e fertilizantes inorgânicos têm demonstrado aumentos na densidade de colêmbolos, quando comparado àqueles solos onde somente utilizam-se fertilizantes inorgânicos (CULIK et al., 2002). Embora esse aumento esteja relacionado à adição de fonte de alimento na forma de esterco e microrganismos que estão juntos.

A espécie Folsomia candida tem sido a mais utilizada em ensaios ecotoxicológicos para avaliar o efeito de diversos compostos como dejetos suínos com e sem antibióticos (doxiciclina e colistina) (MACCARI et al., 2016), lodo de esgoto (DOMENE et al., 2007; NATAL-DA-LUZ et al., 2011), herbicidas (fenmedifan, NATAL-DA-LUZ et al., 2008), fungicidas [(carbendazim, NATAL-DA-LUZ et al., 2008); (captane carboxina + tiram, ALVES et al., 2014)], inseticida [(diazinon, NATAL-DA-LUZ et al., 2012); (cipermetrina, ZORTÉA et al., 2015); (imidacloprida, fipronil, tiametoxam, ALVES et al., 2014)].

Outras espécies de colêmbolos menos utilizadas em testes ecotoxicológicos, como Paronychiurus kimi têm sido bastante úteis para avaliar o risco ecológico do herbicida Paraquat no ecossistema solo, a taxa de sobrevivência dos adultos e a reprodução não foram afetadas pelo Paraquat, exceto na dose mais elevada $\left(1.600 \mathrm{mg} \mathrm{cm}^{-2}\right.$ ) (CHOI et al., 2008). Assim como as espécies Isotoma anglicana, Heteromurus nitidus, Lepidocyrtus violaceus, F. candida e Onychiurus armatus sofreram ação repelente dependente da dose em ensaio de fuga com o herbicida Betanal (HEUPEL 2002).

A exposição à contaminação do inseticida carbofuran causou mudanças significativas na estrutura da comunidade de colêmbolos, com um forte declínio na sua abundância. Foi observada uma relação dose-resposta, mostrando um declínio consistente sobre a abundância relativa de Isotomidae, seguido de perto por um aumento de Entomobryidae. Análise baseada nas características de Collembola sugere que uma alteração na composição funcional das comunidades ocorreu devido à contaminação do solo e que espécies adaptadas para camadas mais profundas do solo eram mais vulneráveis à toxicidade inseticida (CHELINHO et al., 2014).

Colêmbolos mostram diferenças específicas entre espécies na sensibilidade de metais com diminuição na abundância de espécies ao longo de um gradiente de contaminação, enquanto outras mantém ou mesmo aumentam sua população (FILSER et al., 2000; SANTORUFO et al., 2012).

\section{Estudos com fungos}

Os fungos representam uma parte importante da biomassa viva do horizonte superficial e servem como uma importante fonte de alimento 
para os colêmbolos e muitos organismos do solo (BÖLLMANN et al., 2009). Fungos decompositores e FMAs são essenciais para o ótimo crescimento e saúde das plantas, através de seu papel na ciclagem e transporte de nutrientes minerais para as plantas, enquanto que fungos patogênicos de raiz podem trazer grandes perdas em culturas e vegetação natural (LARSEN et al., 2008). Comum a estes fungos é a sua coexistência com a fauna do solo, consequentemente, servir como dieta de diversos invertebrados, incluindo os colêmbolos (LARSEN et al., 2008). As preferências alimentares desses são de interesse para os sistemas agrícolas, pois o pastejo de fungos pode alterar o balanço entre fungos mutualísticos, patogênicos e saprofíticos e o impacto no crescimento de plantas e absorção de nutrientes (SCHREINER; BETHLENFALVAY, 2003).

Micélios fúngicos são fontes de comida altamente nutritivas a muitos invertebrados que se alimentam diretamente do micélio ou esporos, ou indiretamente ingerindo micélio presente na matéria orgânica (BODDY; JONES, 2008). Colêmbolos alimentam-se de micélios fúngicos mantendo constante seu crescimento na fase logarítmica, sendo, assim, positivo para o estoque de nutrientes do solo (PARKINSON et al., 1979). Nas suas fezes, frequentemente são encontrados esporos fúngicos, podendo ser uma explicação para a distribuição de fungos dentro do solo (LUSSENHOP, 1996; VISSER et al., 1987). Espécies de colêmbolos, como Folsomia fimetaria, Hypogastrura assimilis e P. minuta, são capazes de carregar conídios viáveis de fungos entomopatogênicos, como Beauveria bassiana, B. Brongniartii e Metarbizium anisopliae, na cutícula e no intestino (DROMPH, 2001).

Por outro lado, intensa atividade de alimentação pelos colêmbolos podem resultar em uma menor infecção de micorrizas e, consequentemente, diminuir os efeitos de crescimento na planta (HARRIS; BOERNER, 1990; LUSSENHOP, 1996). Experimentos sugerem que a seletividade de colêmbolos é maior em se alimentar de hifas patogênicas do que fungos saprofíticos e maior que fungos micorrízicos (CURL, 1988). Isto pode ser devido aos fungos conterem várias toxinas/compostos repelentes, a fim de evitar o pastejo de hifas ou poder ser um fator de qualidade nutricional dos fungos, já que fungos diferem no seu conteúdo em, por exemplo, carboidratos, proteínas e lipídios (LARSEN et al., 2008). Todavia, os compostos repelentes e as estruturas cristalinas na superfície das hifas são estratégias defensivas de fungos do solo e influenciam fortemente a preferência alimentar de F. candida (BÖLLMANN et al., 2009). Também é possível que os colêmbolos procurem fungos que melhor satisfaçam suas necessidades nutricionais (LARSEN et al., 2008).

Diminuição no crescimento de plantas tem sido mostrada mais frequentemente em plantas micorrizadas e pode resultar do efeito de: (1) pastejo de raízes diretamente por colêmbolos, desta maneira reduzindo a entrada de nutrientes (ENDLWEBER et al., 2009; HARRIS, BOERNER, 1990); (2) pastejo externo de micélio de FMAs reduzindo a entrada de nutrientes (MOORE et al., 1985, WARNOCK et al., 1982); (3) pastejo de micélio de fungos saprofíticos reduz a mineralização da matéria orgânica e liberação de nutrientes (HANLON; ANDERSON, 1979).

Aumentos no crescimento das plantas podem surgir de: (1) pastejo de fungos patogênicos/parasitários reduzindo, assim, a incidência de doença em plantas (CURL et al., 1988); por exemplo, pastejo de Botrytis cinerea (WARDLE, 2002), Gaeumannomyces graminis var. Tritici, Fusarium culmorum, Pythium ultimum e Fusarium oxysporum f. sp. cucumerinum por colêmbolos relatados em diversos autores citados por Sabatini e Innocenti (2001) e Rhizoctonia solani por P. minuta (LARTEY et al., 1994). Além disso, o pastejo de fungos fitopatogênicos pode resultar em uma correlação positiva entre número de colêmbolos e fitomassa (LUSSENHOP; BASSIRIRAD, 2005); (2) pastejo de fungos saprofíticos (vivos ou mortos), por exemplo, Penicillium bordei e Trichoderma harzianum, liberando nutrientes contidos na biomassa fúngica para absorção pelas plantas (INESON et al., 1982; SEASTEDT, 1984). O grupo Collembola também pode afetar o crescimento da planta, aumentando a disponibilidade de nutrientes através da alimentação sobre bactérias e fungos, e mobilização de nutrientes microbianos (ENDLWEBER; SCHEU, 2007). Ainda, (3) o pastejo pode conduzir uma estimulação compensatória na taxa de crescimento fúngico (em FMA ou fungos saprofíticos) e consequentemente uma maior captação de nutrientes por FMA ou aumentos na mineralização e liberação de nutrientes através de fungos saprofíticos (FINLAY, 1985; HEDLUND et al., 1991).

Os colêmbolos vivem e atuam na mesma porção do solo como os fungos e são parte de um complexo de organismos antagônicos e concorrentes que podem influenciar as interações fungo-planta de forma positiva ou negativa. De forma positiva, a alimentação moderada pode manter as micorrizas em um estado ativo, promovendo deste modo o crescimento da planta, bem como limitar o 
crescimento de fungos fitopatogênicos. Ainda, pode promover a proliferação de hifas pela fragmentação e estimular o crescimento das plantas pela liberação de nutrientes. Por outro lado, negativamente, o pastejo excessivo dos colêmbolos sobre as micorrizas pode diminuir a área de influências das hifas e diminuir a infecção. Pode, também, perturbar as conexões de hifas de fungos micorrízicos na medida em que o transporte de água e nutrientes minerais às raízes são diminuídos, e consumo por colêmbolos pode exceder a produção de hifa.

\section{Estudos com ciclagem de nutrientes e fertilidade do solo}

A fauna do solo forma um importante reservatório de nutrientes no solo, como demonstrado por Teuben e Verhoef (1992). Esses autores compararam a concentração de nutrientes $\left(\mathrm{K}^{+}, \mathrm{Ca}^{2+}, \mathrm{Mg}^{2+}, \mathrm{PO}_{4}{ }^{3-}, \mathrm{N}\right.$ e $\left.\mathrm{C}\right)$ entre a serapilheira e alguns grupos de artrópodes (colêmbolos, ácaros oribatídeos, isópodos e diplópodos), mostraram que os nutrientes avaliados estavam concentrados em sua biomassa.

Os colêmbolos são comuns e abundantes em diversos tipos de solos e sistemas de cultivos em todo o mundo, alimentando-se de microrganismos e matéria orgânica em decomposição, com significante influência na ecologia microbiana, ciclagem de nutrientes e fertilidade do solo.

Collembola é um dos principais grupos de invertebrados do solo, porém apresenta baixa biomassa na maioria dos solos, tipicamente menor que $0,2 \mathrm{~g} \mathrm{~m}^{-2}$ comparado aos mais de $20 \mathrm{~g} \mathrm{~m}^{-2}$ apresentado pelas minhocas (CHAMBERLAIN et al., 2006). A contribuição direta deles na respiração do solo e no estoque de $\mathrm{C}$ é pequena, sendo menor que $1 \%$ da respiração total do solo (COULSON; WHITTAKER, 1978).

Grande parte dos solos contém milhões de pelotas fecais de colêmbolos por $\mathrm{m}^{-2}$, que são benéficas, liberando nutrientes essenciais às plantas (HOPKIN, 2002). Contribuem com a decomposição de resíduos de plantas e fungos na superfície e subsuperfície do solo, influenciando no reservatório de $\mathrm{N}$, como também do $\mathrm{C}$, devido a sua mobilidade no substrato e visitar áreas maiores durante a busca por comida (SCHRÖDER, 2008).

A influência direta dos colêmbolos na mineralização da matéria orgânica é subestimada em grande parte dos estudos. Ghilarov (1971) e Van Straalen (1998) calcularam uma quantia de $6 \%$ do total da mineralização da serapilheira em ecossistema de floresta e, com isto, os animais liberam 40 vezes mais nitrogênio que o ambiente vizinho. Ainda, em campos agrícolas foi encontrada correlação positiva entre nitrogênio e abundância de colêmbolos (FILSER et al., 1999). A mobilização de nitrogênio por estas espécies poderia ser importante para muitos ecossistemas, porém é insignificante em solo agrícola fertilizado (SCHRÖDER, 2008). Populações de colêmbolos tendem a imobilizar nitrogênio disponível no solo e o efeito do pastejo de colêmbolos sobre a mineralização de nitrogênio é dependente da espécie. Enquanto uma única espécie apresentou principalmente um efeito de imobilização, a mistura de espécies aumentou a mineralização do nitrogênio (MEBES; FILSER, 1998).

Os colêmbolos podem ter grandes efeitos indiretos no ecossistema do solo. Sua atividade aumenta o carbono orgânico dissolvido e liberação de amônio (COÛTEAUX, et al. 1991; TEUBEN; ROELOFSMA, 1990) e aumenta a mineralização de N (BARDGETT; CHAN, (1999). De acordo com Chamberlain et al. (2006), os colêmbolos são responsáveis pela translocação do $\mathrm{C}$ da serapilheira para o interior do solo e mudanças na composição da matéria orgânica do solo, como também do aumento da transferência do C para a comunidade microbiana do solo. Juntamente com nematóides, os colêmbolos podem aumentar a disponibilidade de nitrato e amônio a plantas pelo consumo de bactérias e fungos na rizosfera (COLEMAN et al., 1983; ELLIOTT et al., 1983; GANGE, 2000; HODGE et al., 2000).

Ilieva-Makulec et al. (2006) encontraram um aumento na abundância e diversidade de colêmbolos em serapilheira de baixa qualidade, com relação C:N inicial alta. Uma possível explicação seria que as frações facilmente degradadas do material sejam cobertas por uma biomassa microbiana ativa, sendo bactérias geralmente observadas no princípio do processo de decomposição (SWIFT et al., 1979). Uma menor biomassa fúngica e uma maior liberação de carbono foram encontradas em tratamentos com colêmbolos juvenis ( $F$. candida), enquanto que a situação inversa ocorreu no tratamento com adultos, indicando que o uso diferenciado de recursos alimentares de acordo com a idade do animal também pode influenciar a decomposição e a ciclagem de nutrientes (BAKONYI, 1989).

O tamanho relativamente grande da população de colêmbolos e a influência potencial na mobilização de nutrientes fazem desses organismos um componente importante na produtividade local e processos em agroecossistemas. 


\section{CONCLUSÃO}

O Brasil possui a maior biodiversidade do planeta e a fauna do solo é um importante componente dessa diversidade. Apesar de ser, na sua maior parte "escondida" por estar no interior do solo ou da serapilheira, os colêmbolos geram importantes serviços ambientais, que são, infelizmente, pouco reconhecidos e valorizados pela Ciência do Solo. Naturalmente, o assunto sobre colêmbolos não se esgota aqui, devido à complexidade do tema e à necessidade de um maior aprofundamento em pesquisas acerca das diversas relações colêmbolosambiente-organismos. A maior prioridade para viabilizar o conhecimento e importância desses organismos está no estímulo para se pesquisar o grupo, além de estudos de diversidade, em nível de espécies e grupos ecomorfológicos, que abordem aspectos positivos e negativos de interações com microrganismos e outros organismos, ciclagem de nutrientes e fertilidade do solo, influência de perturbação e poluição (ecotoxicologia do solo) e a relação da comunidade de colêmbolos com variáveis ambientais explicativas (altitude, temperatura, umidade, diversidade florística, tipo e uso do solo, dentre outras características edáficas) por meio de técnicas de análises multivariadas.

\section{AGRADECIMENTOS}

D. Baretta agradece ao CNPq pela Bolsa de Produtividade Científica (Processo no 307162/2015$0)$.

\section{REFERÊNCIAS}

ABRANTES, E. A.; BELLINI, B. C.; BERNARDO, A. N.; FERNANDES, L. H.; MENDONÇA, M. C.; OLIVEIRA, E. P.; QUEIROZ, G. C.; SAUTTER, K. D.; SILVEIRA, T. C.; ZEPPELINI, D. Errata Corrigenda and update for the "Synthesis of Brazilian Collembola: an update to the species list." ABRANTES et al. (2010), Zootaxa, 2388: 1-22 Zootaxa, v. 3168, p. 1-21, 2012.

ALVAREZ, T.; FRAMPTON, G. K.; GOULSON, D. Epigeic Collembola in winter wheat under organic, integrated and conventional farm management regimes. Agriculture, Ecosystems and Environment, v. 83, n. 1-2, p. 95-110, 2001.
ALVES, P. R. L.; CARDOSO, E. J. B. N.; MARTINES, A. M.; SOUSA, J. P.; PASINI, A. Seed dressing pesticides on springtails in two ecotoxicological laboratory tests. Ecotoxicology and Environmental Safety, v. 105, n. 1, p. 65-71, 2014.

BAKONYI, G. Effects of Folsomia candida (Collembola) on the microbial biomass in a grassland soil. Biology and Fertility of Soils, v. 7, n. 2, p. 138141, 1989.

BAKONYI, G.; POSTA, K.; KISS, I.; FÁBIÁN, M.; NAGY, P.; NOSEK, J. N. Density-dependent regulation of arbuscular mycorrhiza by collembola. Soil Biology and Biochemistry, v. 34, n. 5, p. 661-664, 2002.

BARDGETT, R. D.; CHAN, K. F. Experimental evidence that soil fauna enhance nutrient mineralization and plant nutrient uptake in montane grassland ecosystems. Soil Biology and Biochemistry, v. 31, p. 1007-1014, 1999.

BARETTA, D.; FERREIRA, C. S.; SOUSA, J. P.; CARDOSO, E. J. B. N. Colêmbolos (Hexapoda: Collembola) como bioindicadores de qualidade do solo em áreas com Araucaria angustifolia. Revista Brasileira de Ciência do Solo, v. 32, n. spe, p. 26932699, 2008.

BARETTA, D.; MAFRA, Á. L.; SANTOS, J. C. P.; DO AMARANTE, C. V. T.; BERTOL, I. Análise multivariada da fauna edáfica em diferentes sistemas de preparo e cultivo do solo. Pesquisa Agropecuária Brasileira, v. 41, n. 11, p. 1675-1679, 2006.

BARETTA, D.; SANTOS, J. C. P.; MAFRA, Á. L.; WILDNER, L. P.; MIQUELLUTI, D. J. Fauna edáfica avaliada por armadilhas de catação manual afetada pelo manejo do solo na região oeste catarinense. Revista de Ciências Agroveterinárias, v. 2, p. $97-106,2003$.

BARROS, Y. J.; MELO, V. D. F.; SAUTTER, K. D.; et al. Indicadores de qualidade de solos de área de mineração e metalurgia de chumbo. II - Mesofauna e plantas. Revista Brasileira de Ciência do Solo, v. 34, n. 4, p. 1413-1426, 2010.

BARTZ, M. L. C.; BROWN, G. G.; ORSO, R.; MAFRA, Á. L.; BARETTA, D. The influence of land use systems on soil and surface litter fauna in the 
western region of Santa Catarina. Revista Ciência Agronômica, v. 45, n. 5 (Especial), p. 880-887, 2014.

BELLINGER, P. F.; CHRISTIANSEN, K. A.; JANSSENS, F. Checklist of the Collembola of the World. Disponível

em: $<$ http://www.collembola.org $>$. Acesso em: 01 mar. 2016.

BENGTSSON, J. Disturbance and resilience in soil animal communities. European Journal of Soil Biology, v. 38, p. 119-125, 2002.

BERBIERS, P.; MAELFAIT, J. P.; MERTENS, J. Evaluation of some sampling methods used to study Collembola (Insecta, Apterygota) in a pasture. Revue d'Écologie et de Biologie du Sol, v. 26, n. 3, p. 305$320,1989$.

BODDY, L.; JONES, T. H. Ecology of Saprotrophic Basidiomycetes. Amsterdam: Elsevier, 2008.

BÖLLMANN, J.; ELMER, M.; WÖLLECKE, J.; RAIDL, S.; HÜTTL, R. F. Defensive strategies of soil fungi to prevent grazing by Folsomia candida (Collembola). Pedobiologia, v. 53, n. 2, p. 107-114, 2009.

BRENNAN, A.; FORTUNE, T.; BOLGER, T. Collembola abundances and assemblage structures in conventionally tilled and conservation tillage arable systems. Pedobiologia, v. 50, p. 135-145, 2006.

BREVIK, E. C.; CERDÀ, A.; MATAIX-SOLERA, J.; PEREG, L.; QUINTON, J. N.; SIX, J.; VAN OOST, K. The interdisciplinary nature of SOIL. Soil, v. 1, n. 1, p. 117-129, 2015.

BRONICK, C. J.; LAL, R. Soil structure and management: a review. Geoderma, v. 124, n. 1-2, p. 322, 2005.

BROWN, G. G.; NIVA, C. C.; ZAGATTO, M. R. G.; Ferreira, S. S.; Nadolny, H. S.; Cardoso, G. B. X.; Santos, A.; Martinez, G .A.; Pasini, A.; Bartz, M. L. C.; Sautter, K. D.; Thomazini, M. J.;Baretta, D.; Silva, E.; Antoniolli, Z. I.; Decaëns, T.; Lavelle, P. M.; Sousa, J. P.; Carvalho, F. Biodiversidade da fauna do solo e sua contribuição para os serviços ambientais. In: PARRON, L. M.; GARCIA, J. R.; OLIVEIRA, E. B.; BROWN, G. G.; PRADO, R. B. (Orgs.) Serviços Ambientais em Sistemas Agrícolas e Florestais do
Bioma Mata Atlântica. $1^{a}$ ed., Brasília: Embrapa, 2015, p. $122-154$.

BUZZI, Z. J. Entomologia Didática. Curitiba: Editora UFPR, 2013.

CARUSO, T.; BARTO, E. K.; SIDDIKY, M. R. K.; SMIGELSKI, J.; RILLIG, M. C. Are power laws that estimate fractal dimension a good descriptor of soil structure and its link to soil biological properties? Soil Biology and Biochemistry, v. 43, n. 2, p. 359-366, 2011.

CASSAGNE, N.; GERS, C.; GAUQUELIN, T. Relationships between Collembola, soil chemistry and humus types in forest stands (France). Biology and Fertility of Soils, v. 37, n. 6, p. 355-361, 2003.

CHAGNON, M.; PARÉ, D.; HÉBERT, C. Relationships between soil chemistry, microbial biomass and the collembolan fauna of southern Québec sugar maple stads. Ecoscience, v. 7, n. 3, p. 307-316, 2000.

CHAMBERLAIN, P. M.; MCNAMARA, N. P.; CHAPLOW, J.; STOTT, A. W.; BLACK, H. I. J. Translocation of surface litter carbon into soil by Collembola. Soil Biology and Biochemistry, v. 38, p. 2655-2664, 2006.

CHAUVAT, M.; WOLTERS, V.; DAUBER, J. Response of collembolan communities to land-use change and grassland succession. Ecography, v. 30, p. 183-192, 2007.

CHAUVAT, M.; ZAITSEV, A. S.; WOLTERS, V. Successional changes of Collembola and soil microbiota during forest rotation. Oecologia, v. 137, n. 2, p. 269-276, 2003.

CHELINHO, S.; DOMENE, X.; ANDRÉS, P.; Natal-DA-LUZ, T.; NORTE, C.; RUFINO, C.; LOPES, I.; CACHADA, A.; ESPÍNDOLA, E.; RIBEIRO, R.; DUARTE, A. C.; SOUSA, J. P. Soil microarthropod community testing: A new approach to increase the ecological relevance of effect data for pesticide risk assessment. Applied Soil Ecology, v. 83, p. 200-209, 2014.

CHOI, W. IL; NEHER, D. A.; RYOO, M. IL. Lifehistory trade-offs of Paronychiurus kimi (Lee) (Collembola: Onychiuridae) populations exposed to 
paraquat. Ecotoxicology and environmental safety, v. 69 , n. 2 , p. $227-232,2008$.

CHRISTIANSEN, K. Bionomics of Collembola. Annual Review of Entomology, v. 9, n. 1, p. 147-178, 1964.

CHRISTIANSEN, K. Survival of Collembola on clay substrates with and without food added. Annales de Spéléologie, v. 25, n. 4, p. 849-852, 1970.

CLUZEAU, D.; GUERNION, M.; CHAUSSOD, R.; MARTIN-LAURENT, F.; VILLENAVE， C.; CORTET, J.; RUIZ-CAMACHO, N.; PERNIN, C.; MATEILLE, T.; PHILIPPOT, L.; BELLIDO, A.; ROUGÉ, L.; ARROUAYS, D.; BISPO, A.; PÉRÈS, G. Integration of biodiversity in soil quality monitoring: Baselines for microbial and soil fauna parameters for different land-use types. European Journal of Soil Biology, v. 49, p. 63-72, 2012.

COLEMAN, D. C.; REID, C. P. P.; COLE, C. V. Biological Strategies of Nutrient Cycling in Soil Systems. In: MACFADYEN, A.; FORD, E. D. (Orgs.) Advances in Ecological Research, Advances in Ecological Research. London: Academic Press., v. 13, 1983, p. 1-55.

COULSON, J. C.; WHITTAKER, J. B. Ecology of moorland animals. In: HEAL, O. W.; PERKINS, D. F. (Orgs.) Production Ecology of British Moors and Montane Grasslands. Berlin: Springer-Verlag. 1978, p. 52-93.

COÛTEAUX, M.-M.; MOUSSEAU, M.; CÉLÉRIER, M.-L.; BOTTNER, P. Increased atmospheric $\mathrm{CO}^{2}$ and litter quality: decomposition of sweet Chestnut leaf litter with animal food webs of different complexities. Oikos, v. 61, n. 1, p. 54-64, 1991.

CULIK, M. P.; MARTINS, D. D. S.; VENTURA, J. A. Collembola (Arthropoda: Hexapoda) communities in the soil of papaya orchards managed with conventional and integrated production in Espírito Santo, Brazil. Biota Neotropica, v. 6, n. 3, 2006.

CULIK, M. P.; SOUZA, J. L.; VENTURA, J. A. Biodiversity of Collembola in tropical agricultural environments of Espírito Santo, Brazil. Applied Soil Ecology, v. 21, p. 49-58, 2002.
CURL, E. A. The role of soil microfauna in plantdisease suppression. Critical Reviews in Plant Sciences, v. 7, n. 3, p. 175-196, 1988.

CURL, E. A.; LARTEY, R.; PETERSON, C. M. Interactions between root pathogens and soil microarthropods. Agriculture, Ecosystems and Environment, v. 24, n. 1-3, p. 249-261, 1988.

DALY, H. V; DOYEN, J. T.; PURCELL, A. H. Introduction to Insect Biology and Diversity. New York: Oxford University Press, 1998.

DAVIDSON, D. A.; GRIEVE, I. C. Relationships between biodiversity and soil structure and function: Evidence from laboratory and field experiments. Applied Soil Ecology, v. 33, n. 2, p. 176-185, 2006.

DIDDEN, W. A. M. Reactions of Onychiurus fimatus (Collembole) to loose and compact soil. Method and first results. Pedobiologia, v. 30, p. 93-100, 1987.

DITTMER, S.; SCHRADER, S. Longterm effects of soil compaction and tillage on Collembola and straw decomposition in arable soil. Pedobiologia, v. 44, p. 527-538, 2000.

DOMENE, X.; ALCAÑIZ, J. M.; ANDRÉS, P. Ecotoxicological assessment of organic wastes using the soil collembolan Folsomia candida. Applied Soil Ecology, v. 35, n. 3, p. 461-472, 2007.

DRESCHER, M. S.; ROVEDDER, A. P. M.; ANTONIOLLI, Z. I.; ELTZ, F. L. F.; DRESCHER, G. L. Fauna epigeica em sistemas de produção de Nicotiana tabacum L. Revista Brasileira de Ciência do Solo, v. 35, n. 4, p. 1499-1507, 2011.

DUARTE, M. M. Abundância de microartrópodes do solo em fragmentos de mata com araucária no sul do Brasil. Iheringia, Série Zoologia, v. 94, n. 2, p. 163169, 2004.

EATON, R. J.; BARBERCHECK, M.; BUFORD, M.; SMITH, W. Effects of organic matter removal, soil compaction, and vegetation control on Collembolan populations. Pedobiologia, v. 48, n. 2, p. 121-128, 2004.

ELLIOTT, E. T.; HORTON, K.; MOORE, J. C.; COLEMAN, D. C.; COLE, D. C. Mineralization dynamics in fallow dryland wheat plots, Colorado. Plant and Soil, v. 76, p. 149-155, 1983. 
ENDLWEBER, K.; RUESS, L.; SCHEU, S. Collembola switch diet in presence of plant roots thereby functioning as herbivores. Soil Biology and Biochemistry, v. 41, n. 6, p. 1151-1154, 2009.

ENDLWEBER, K.; SCHEU, S. Interactions between mycorrhizal fungi and Collembola: effects on root structure of competing plant species. Biology and Fertility of Soils, v. 43, n. 6, p. 741-749, 2007.

FILSER, J.; DETTE, A.; FROMM, H.; et al. Reactions of soil organisms to site-specific management: The first long-term study at the landscape level. Ecosystem, v. 28, p. 139-147, 1999.

FILSER, J.; WITTMANN, R.; LANG, A. Response types in Collembola towards copper in the microenvironment. Environmental Pollution, v. 107, n. 1, p. 71-78, 2000.

FINLAY, R. D. Interactions between soil microarthropods and endomycorrhizal associations of higher plants. In: FITTER, A. H.; ATKINSON, D.; READ, D. J.; USHER, M. B. (Orgs.) Ecological interactions in soil. Oxford: Blackwell Scientific Publications. 1985, p. 319-331.

FOUNTAIN, M. T.; HOPKIN, S. P. Folsomia candida (Collembola): A "Standard" Soil Arthropod. Annual Review of Entomology, v. 50, p. 201-222, 2005.

FRAMPTON, G. K.; VAN DEN BRINK, P. J. Collembola and macroarthropod community responses to carbamate, organophosphate and synthetic pyrethroid insecticides: Direct and indirect effects. Environmental Pollution, v. 147, p. 14-25, 2007.

GAMA, M. M.; SOUSA, J. P.; VASCONCELOS, T. M.; FERREIRA, C. S.; BARROCAS, H. Changes in biodiversity patterns of soil Collembola caused by eucalyptus plantations in Portugal: a synthesis. Acta Entomológica Ibérica e Macaronésica, v. 1, p. 11-22, 2003.

GANGE, A. Arbuscular mycorrhizal fungi, Collembola and plant growth. Trends in Ecology \& Evolution, v. 15, n. 9, p. 369-372, 2000.

GANGE, A. C.; BOWER, E. Interactions between insects and mycorrhizal fungi. In: GANGE, A. C.; BROWN, V. K. (Orgs.) Multitrophic Interactions in
Terrestrial Systems. Oxford: Blackwell Science, 1997, p. 115-132.

GARCÍA-GÓMEZ, A.; CASTAÑO-MENESES, G.; PALACIOS-VARGAS, J. G. Diversity of springtails (Hexapoda) according to a altitudinal gradient. Pesquisa Agropecuária Brasileira, v. 44, n. 8, p. 911916, 2009.

GHILAROV, M. S. Invertebrates which destroy the forest litter and ways to increase their activity. Ecology and Conservation, v. 4, p. 433-439, 1971.

GILLER, P. S. The diversity of soil communities, the "poor man"s tropical rainforest'. Biodiversity and Conservation, v. 5, n. 2, p. 135-168, 1996.

GISIN, H. Ökologie und Lebensgemeinschaften der Collembolen im schweizerischen Exkursionsgebiet Basels. Revue Suisse de Zoologie, v. 50, n. 1943, p. 131-224, 1943.

GRONENBERG, W. The trap-jaw mechanism in the dacetine ants Daceton armigerum and Strumigenys sp. The Journal of Experimental Biology, v. 199, p. 20212033, 1996.

HAARLØV, N. Microarthropods from Danish Soils : Ecology, Phenology. Copenhagen: Munksgaard, 1960.

HANLON, R. D. G.; ANDERSON, J. M. The effects of collembola grazing on microbial activity in decomposing leaf litter. Oecologia, v. 38, n. 1, p. 9399, 1979.

HARRIS, K. K.; BOERNER, R. E. J. Effects of belowground grazing by collembola on growth, mycorrhizal infection, and $\mathrm{P}$ uptake of Geranium robertianum. Plant and Soil, v. 129, p. 203-210, 1990.

HAVLICEK, E. Soil biodiversity and bioindication: From complex thinking to simple acting. European Journal of Soil Biology, v. 49, p. 80-84, 2012.

HEDLUND, K.; BODDY, L.; PRESTON, C. M. Mycelial responses of the soil fungus, Mortierella isabellina, to grazing by Onychiurus armatus (Collembola). Soil Biology and Biochemistry, v. 23, n. 4, p. 361-366, 1991.

HEISLER, C.; KAISER, E.-A. Influence of agricultural traffic and crop management on 
collembola and microbial biomass in arable soil. Biology and Fertility of Soils, v. 19, p. 159-165, 1995.

HEUPEL, K. Avoidance response of different collembolan species to Betanal. European Journal of Soil Biology, v. 38, n. 3-4, p. 273-276, 2002.

HODGE, A.; STEWART, J.; ROBINSON, D.; GRIFFITHS, B. S.; FITTER, A. H. Plant N capture and microfaunal dynamics from decomposing grass and earthworm residues in soil. Soil Biology and Biochemistry, v. 32, n. 11-12, p. 1763-1772, 2000.

HODKINSON, I. D.; HEALEY, V.; COULSON, S. Moisture relationships of the high arctic collembolan Onychiurus arcticus. Physiological Entomology, v. 19, n. 2, p. 109-114, 1994.

HOPKIN, S. P. Biology of the Springtails (Insecta: Collembola). Oxford: Oxford University Press, 1997.

HOPKIN, S. P. Collembola: the most abundant insects on earth. Antenna, v. 22, p. 117-121, 1998.

HOPKIN, S. P. Collembola. In: LAL R. Encyclopedia of Soil Science. New York: Marcel Dekker, 2002, p. 207-210.

HUTCHINS, M.; EVANS, A. V; GARRISON, R. W.; SCHLAGER, N. Insects. In: GRZIMEK, B. Grzimek's Animal Life Encyclopedia. Farmington Hills: Gale Group, 2004, p. 99-105.

ILIEVA-MAKULEC， K.; OLEJNICZAK， I.; SZANSER, M. Response of soil micro- and mesofauna to diversity and quality of plant litter. European Journal of Soil Biology, v. 42, p. S244S249, 2006.

INESON, P.; LEONARD, M. A.; ANDERSON, J. M. Effect of collembolan grazing upon nitrogen and cation leaching from decomposing leaf crop residue. Soil Biology and Biochemistry, v. 14, p. 601-605, 1982.

JANSSENS, F.; CHRISTIANSEN, K. A. Class Collembola Lubbock, 1870. In: Zhang, Z.-Q. Animal biodiversity: An outline of higher-level classification and survey of taxonomic richness (Zootaxa 3148). Auckland: Magnolia Press., 2011, p.192-194.

JOOSSE, E. N. G. Ecological strategies and population regulation of Collembola in heterogeneous environments. Pedobiologia, v. 21, p. 346-356, 1981.

JØRGENSEN, H. B.; ELMHOLT, S.; PETERSEN, $\mathrm{H}$. Collembolan dietary specialisation on soil grown fungi. Biology and Fertility of Soils, v. 39, n. 1, p. 915, 2003.

JUCEVICA, E.; MELECIS, V. Global warming affect Collembola community: A long-term study. Pedobiologia, v. 50, n. 2, p. 177-184, 2006.

KLADIVKO, E. J. Tillage systems and soil ecology. Soil \& Tillage Research, v. 61, n. 1-2, p. 61-76, 2001.

KRAB, E. J.; OORSPRONG, H.; BERG, M. P.; CORNELISSEN, J. H. C. Turning northern peatlands upside down: Disentangling microclimate and substrate quality effects on vertical distribution of Collembola. Functional Ecology, v. 24, n. 6, p. $1362-$ 1369, 2010.

LARSEN, J.; JOHANSEN, A.; LARSEN, S. E.; et al. Population performance of collembolans feeding on soil fungi from different ecological niches. Soil Biology and Biochemistry, v. 40, n. 2, p. 360-369, 2008.

LARSEN, T.; SCHJØNNING, P.; AXELSEN, J. The impact of soil compaction on euedaphic Collembola. Applied Soil Ecology, v. 26, p. 273-281, 2004.

LARTEY, R. T.; CURL, E. A.; PETERSON, C. M. Interactions of mycophagous collembola and biocontrol fungi in the suppression of Rhizoctonia solani. Soil Biology and Biochemistry, v. 26, n. 1, p. 81-88, 1994.

LAVELLE, P.; BIGNELL, D.; LEPAGE, M.; et al. Soil function in a changing world: the role of invertebrate ecosystem engineers. European Journal of Soil Biology, v. 33, p. 159-193, 1997.

LUSSENHOP, J. Collembola as mediators of microbial symbiont effects upon soybean. Soil Biology and Biochemistry, v. 28, n. 3, p. 363-369, 1996.

LUSSENHOP, J.; BASSIRIRAD, H. Collembola effects on plant mass and nitrogen acquisition by ash seedlings (Fraxinus pennsylvanica). Soil Biology and Biochemistry, v. 37, n. 4, p. 645-650, 2005. 
MACCARI, A. P.; BARETTA, D.; PAIANO, D.; et al. Ecotoxicological effects of pig manure on Folsomia candida in subtropical Brazilian soils. Journal of Hazardous Materials, v. 314, p. 113-120, 2016.

MEBES, K. H.; FILSER, J. Does the species composition of Collembola affect nitrogen turnover? Applied Soil Ecology, v. 9, n. 1, p. 241-247, 1998.

MOORE, J. C.; ST. JOHN, T. V; COLEMAN, D. C. Ingestion of vesicular-arbuscular mycorrhizal hyphae and spores by soil arthropods. Ecology, v. 66, n. 6, p. 1979-1981, 1985.

NAKAMORI, T.; SUZUKI, A. Surface properties of the mushroom Strobilurus obshimae result in food differentiation by collembolan species. European Journal of Soil Biology, v. 44, n. 5-6, p. 478-482, 2008.

NATAL-DA-LUZ, T.; AMORIM, M. J. B.; RÖMBKE, J.; PAULO SOUSA, J. Avoidance tests with earthworms and springtails: Defining the minimum exposure time to observe a significant response. Ecotoxicology and Environmental Safety, v. 71 , n. 2, p. 545-551, 2008.

NATAL-DA-LUZ，T.; MOREIRA-SANTOS， M.; RUEPERT, C.; CASTILLO, L. E.; RIBEIRO, R.; SOUSA, J. P. Ecotoxicological characterization of a tropical soil after diazinon spraying. Ecotoxicology, v. 21, n. 8, p. 2163-2176, 2012.

NATAL-DA-LUZ, T.; OJEDA, G.; PRATAS, J.; VAN GESTEL, C. A. M.; SOUSA, J. P. Toxicity to Eisenia andrei and Folsomia candida of a metal mixture applied to soil directly or via an organic matrix. Ecotoxicology and Environmental Safety, v. 74, n. 6, p. $1715-1720,2011$.

NIETSCHKE, L.; BURFEINDT, I.; SEUPT, A.; FILSER, J. Collembola and seed germination: relevance of substrate quality and evidence for seed attack. Soil Organisms, v. 83, n. 3, p. 451-462, 2011.

PARISI, V.; MENTA, C.; GARDI, C.; JACOMINI, C.; MOZZANICA, E. Microarthropod communities as a tool to assess soil quality and biodiversity: a new approach in Italy. Agriculture, Ecosystems and Environment, v. 105, n. 1-2, p. 323-333, 2005.
PARKINSON, D.; VISSER, S.; WHITTAKER, J. B. Effects of collembolan grazing on fungal colonization of leaf litter. Soil Biology and Biochemistry, v. 11, n. 5, p. 529-535, 1979.

PETERSEN, H. General aspects of collembolan ecology at the turn of the millennium. Pedobiologia, v. 46, p. 246-260, 2002.

PETERSEN, H.; LUXTON, M. A comparative analysis of soil fauna populations and their role in decomposition processes. Oikos, v. 39, p. 288-389, 1982.

PEY, B.; NAHMANI, J.; AUCLERC, A.; CAPOWIEZ, Y.; CLUZEAU, D.; CORTET, J.; DECAËNS, T.; DEHARVENG, L.; DUBS, F.; JOIMEL, S.; BRIARD, C.; GRUMIAUX, F.; LAPORTE, M. A.; PASQUET, A.; PELOSI, C.; PERNIN, C.; PONGE, J. F.; SALMON, S.; SANTORUFO, L.; HEDDE, M. Current use of and future needs for soil invertebrate functional traits in community ecology. Basic and Applied Ecology, v. 15, n. 3, p. 194-206, 2014.

PFLUG, A.; WOLTERS, V. Collembola Communities along a European Transect. European Journal of Soil Biology, v. 38, p. 301-304, 2002.

PONGE, J. F.; GILLET, S.; DUBS, F.; FEDOROFF, E.; HAESE, L.; SOUSA, J. P.; LAVELLE, P. Collembolan communities as bioindicators of land use intensification. Soil Biology and Biochemistry, v. 35, n. 6, p. 813-826, 2003.

PONGE, J.-F.; DUBS, F.; GILLET, S.; SOUSA, J. P.; LAVELLE, P. Decreased biodiversity in soil springtail communities: the importance of dispersal and land use history in heterogeneous landscapes. Soil Biology and Biochemistry, v. 38, p. 1158-1161, 2006.

QUERNER, P.; BRUCKNER, A.; DRAPELA, T.; MOSER, D.; ZALLER, J. G.; FRANK, T. Landscape and site effects on Collembola diversity and abundance in winter oilseed rape fields in eastern Austria. Agriculture, Ecosystems and Environment, v. 164, p. 145-154, 2013.

ROBERTS, J. M. K.; WEEKS, A. R.; HOFFMANN, A. A.; UMINA, P. A. Does Bdellodes lapidaria (Acari: Bdellidae) have a role in biological control of the springtail pest, Sminthurus viridis (Collembola: 
Sminthuridae) in south-eastern Australia? Biological Control, v. 58, n. 3, p. 222-229, 2011.

ROTHERAY, T. D.; BODDY, L.; JONES, T. H. Collembola foraging responses to interacting fungi. Ecological Entomology, v. 34, n. 1, p. 125-132, 2009.

ROVEDDER, A. P. M.; ELTZ, F. L. F.; DRESCHER, M. S.; SCHENATO, R. B.; ANTONIOLLI, Z. I. Organismos edáficos como bioindicadores da recuperação de solos degradados por arenização no Bioma Pampa. Ciência Rural, v. 39, n. 4, p. 1061-1068, 2009.

ROŹEN, A.; SOBCZYK, Ł.; LISZKA, K.; WEINER, J. Soil faunal activity as measured by the bait-lamina test in monocultures of 14 tree species in the Siemianice common-garden experiment, Poland. Applied Soil Ecology, v. 45, p. 160-167, 2010.

RUSEK, J.; MARSHALL, V. G. Impacts of airborne pollutants on soil fauna. Annual Review of Ecology and Systematics, v. 31, p. 395-423, 2000.

SABATINI, M. A.; INNOCENTI, G. Effects of collembola on plant-pathogenic fungus interactions in simple experimental systems. Biology and Fertility of Soils, v. 33, n. 1, p. 62-66, 2001.

SANTORUFO, L.; CAROTENUTO, R.; ROCCO, A.; PICIONE, F. D. L.; MAISTO, G. Orthonychiurus pseudostachianus (collembola) as a toxicity test organism and selection of an ecotoxicological test battery to assess soil quality. Applied Soil Ecology, v. 54, p. 4954, 2012.

SCHRADER, S.; LINGNAU, M. Influence of soil tillage and soil compaction on microarthropods in agricultural land. Pedobiologia, v. 41, p. 202-209, 1997.

SCHREINER, R. P.; BETHLENFALVAY, G. J. Crop residue and Collembola interact to determine the growth of mycorrhizal pea plants. Biology and Fertility of Soils, v. 39, n. 1, p. 1-8, 2003.

SCHRÖDER, P. Mesofauna. In: SCHRÖDER, P.; PFADENHAUER, J.; MUNCH, J. C. (Orgs.) Perspectives for Agroecosystem Management balancing environmental and socioeconomic demands. Amsterdam: Elsevier. 2008, p.293-306.
SEASTEDT, T. R. The role of microarthropods in decomposition and mineralization processes. Annual Review of Entomology, v. 29, p. 25-46, 1984.

SIDDIKY, M. R. K.; SCHALLER, J.; CARUSO, T.; RILLIG, M. C. Arbuscular mycorrhizal fungi and collembola non-additively increase soil aggregation. Soil Biology and Biochemistry, v. 47, p. 93-99, 2012.

SOUSA, J. P.; BOLGER, T.; GAMA, M. M.; LUKKARI, T.; PONGE, J.-F.; SIMÓN, C.; TRASER, G.; VANBERGEN, A. J.; BRENNAN, A.; DUBS, F.; IVITIS, E.; KEATING, A.; STOFER, S.; WATT, A. D. Changes in Collembola richness and diversity along a gradient of land-use intensity: A pan European study. Pedobiologia, v. 50, p. 147-156, 2006.

STINNER, B.; HOUSE, G. Arthropods and other invertebrates in conservation-tillage agriculture. Annual Review of Entomology, v. 35, n. 1, p. 299318, 1990.

VAN STRAALEN, N. M. Evaluation of bioindicator systems derived from soil arthropod communities. Applied Soil Ecology, v. 9, n. 1-3, p. 429-437, 1998.

VAN STRAALEN, N. M.; TIMMERMANS, M. J. T. N.; ROELOFS, D.; BERG, M. P. Apterygota in the spotlights of ecology, evolution and genomics. European Journal of Soil Biology, v. 44, n. 5-6, p. 452-457, 2008.

SWIFT, M. J.; HEAL, O. W.; ANDERSON, J. M. Decomposition in Terrestrial Ecosystems. Oxford: Blackwell Scientific Publications, 1979.

TEUBEN, A.; ROELOFSMA, T. Dynamic interactions between functional groups of soil arthropods and microorganisms during decomposition of coniferous litter in microcosm experiments. Biology and Fertility of Soils, v. 9, n. 2, p. 145-151, 1990.

TEUBEN, A.; VERHOEF, H. A. Direct contribution by soil arthropods to nutrient availability through body and faecal nutrient content. Biology and Fertility of Soils, v. 14, p. 71-75, 1992.

VISSER, S.; PARKINSON, D.; HASSALL, M. Fungi associated with Onychiurus subtenuis (Collembola) in an aspen woodland. Canadian Journal of Botany, v. 65, n. 4, p. 635-642, 1987. 
WAGG, C.; BENDER, S. F.; WIDMER, F.; VAN DER HEIJDEN, M. G. A. Soil biodiversity and soil community composition determine ecosystem multifunctionality. Proceedings of the National Academy of Sciences of the United States of America, v. 111, n. 14, p. 5266-5270, 2014.

WARDLE, D. A. Communities and Ecosystems: Linking the Aboveground and Belowground Components. Princeton: Princeton University Press, 2002.

WARNOCK, A. J.; FITTER, A. H.; USHER, M. B. The influence of a springtail Folsomia candida (Insecta, Collembola) on the mycorrhizal association of leek Allium porrum and the vesicular-arbuscular endophyte Glomus fasciculatus. New Phytologyst, v. 90, n. 2, p. 285-292, 1982.

WINTER, J. P.; VORONEY, R. P.; AINSWORTH, D. A. Soil microarthropods in long-term no-tillage and conventional tillage corn production. Canadian Journal of Soil Science, v. 70, p. 641-653, 1990.

ZEPPELINI, D. Collembola. In: RAFAEL, J. A.; MELO, G. A. R.; CARVALHO, C. J. B.; CASARI, S. A.; CONSTANTINO, R. Insetos do Brasil: Diversidade e Taxonomia. Ribeirão Preto: Holos, 2010, p. 201-212.

ZEPPELINI-FILHO, D.; BELLINI, B. C. Introdução ao estudo dos Collembola. João Pessoa: Universidade Federal da Paraíba, 2004.

ZEPPELINI, D.; BELLINI, B. C.; CREÃODUARTE, A. J.; HERNÁNDEZ, M. I. M. Collembola as bioindicators of restoration in mined sand dunes of Northeastern Brazil. Biodiversity and Conservation, v. 18, n. 5, p. 1161-1170, 2009.

ZHANG, Z.-Q. Animal biodiversity: An outline of higher-level classification and survey of taxonomic richness (Zootaxa 3148). 1o ed. Auckland: Magnolia Press, 2011.

ZORTÉA, T.; BARETTA, D.; MACCARI, A. P.; et al. Influence of cypermethrin on avoidance behavior, survival and reproduction of Folsomia candida in soil. Chemosphere, v. 122, p. 94-98, 2015. 